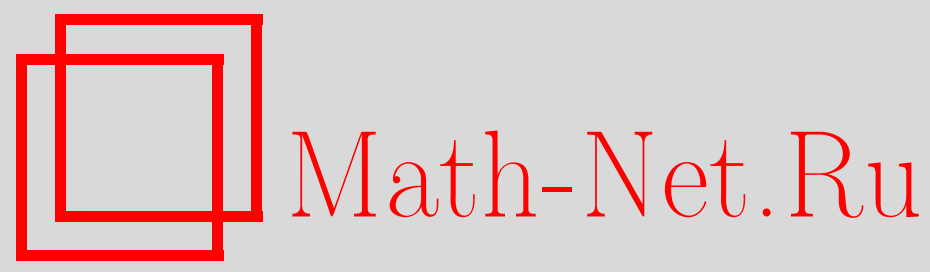

В. В. Смагин, Оценки в сильных нормах погрешности проекционно-разностного метода приближенного решения абстрактного параболического уравнения, Матем. заметки, 1997, том 62, выпуск 6, 898-909

DOI: https://doi.org/10.4213/mzm1679

Использование Общероссийского математического портала Math-Net.Ru подразумевает, что вы прочитали и согласны с пользовательским соглашением http://www . mathnet.ru/rus/agreement

Параметры загрузки:

IP : 3.85 .73 .92

26 апреля 2023 г., 11:33:08

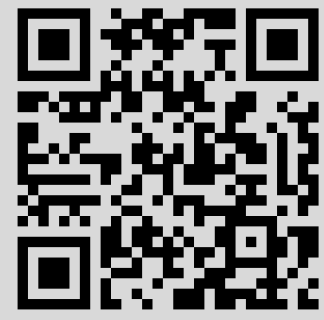




\title{
ОЦЕНКИ В СИЛЬНЫХ НОРМАХ ПОГРЕШНОСТИ ПРОЕКЦИОННО-РАЗНОСТНОГО МЕТОДА ПРИБЛИЖЕННОГО РЕШЕНИЯ АБСТРАКТНОГО ПАРАБОЛИЧЕСКОГО УРАВНЕНИЯ
}

\section{В. В. Смагин}

\begin{abstract}
Непрерьвно дифференцируемые по времени решения параболического уравнения в гильбертовом пространстве отыскиваются проекционно-разностным методом приближенно. Дискретизация задачи по пространству проводится методом Галёркина, а по времени - неявньм методом Эйлера. В работе установлены оценки в сильных нормах погрештости приближенных решений. Эти оценки позволяют получать не только сходимость приближенных решений к точному, но и дают числовые характеристики скорости сходимости. В частности, в работе получены точные по порядку аппроксимации оценки погрешности для подпространств типа конечных элементов.

Библиография: 10 названий.
\end{abstract}

1. Обсуждение задачи и вспомогательные результаты. В сепарабельном гильбертовом пространстве $H$ на отрезке $[0, T]$ рассматривается задача

$$
u^{\prime}(t)+A(t) u(t)+B(t) u(t)=f(t), \quad u(0)=u^{0} .
$$

Предполагается, что оператор $A(t)$ при каждом $t \in[0, T]$ является самосопряженным положительно определенным с областью определения $D[A(t)]$. Без ограничения общности считаем, что

$$
(A(t) u, u) \geqslant\|u\|^{2}, \quad t \in[0, T], \quad u \in D[A(t)],
$$

где $(\cdot, \cdot)$ и $\|\cdot\|$ - скалярное произведение и норма в $H$ соответственно. Для множества $D[A(t)]$ допускается зависимость от $t$. Считаем, однако, что у положительно определенных самосопряженных операторов $A^{1 / 2}(t)$ области определения $D\left[A^{1 / 2}(t)\right]=D_{1 / 2}$ от $t$ не зависят.

Через $V$ обозначим энергетическоепространство, порожденное оператором $A(0)$, т.е.

$$
V=\left\{u \in D_{1 / 2} \mid\|u\|_{V}=\left\|A^{1 / 2}(0) u\right\|\right\} .
$$

Для $u, v \in V$ определим форму

$$
a(t, u, v)=\left(A^{1 / 2}(t) u, A^{1 / 2}(t) v\right) .
$$


Пусть при всех $t \in[0, T]$ и $u, v \in V$ выполнено

$$
|a(t, u, v)| \leqslant M_{1}\|u\|_{V} \cdot\|v\|_{V}, \quad a(t, u, u) \geqslant \delta\|u\|_{V}^{2}, \quad \delta>0
$$

функция $a(t, u, v)$ дифференцируема по $t$ и справедлива оценка

$$
\left|\frac{\partial}{\partial t} a(t, u, v)\right| \leqslant M_{2}\|u\|_{V} \cdot\|v\|_{V}
$$

Предполагается также существование гильбертова пространства $E$ такого, что для всех $t \in[0, T]$ вьполнено $D[A(t)] \subset E \subset V \subset H$, причем пространство $V$ совпадает с точностью до эквивалентной нормы с интерполящионным пространством $[E, H]_{1 / 2}$ (см. $[1$, с. 23]) и для всех $t \in[0, T]$ справедлива оценка

$$
\|u\|_{E} \leqslant d\|A(t) u\|, \quad u \in D[A(t)] .
$$

Заметим, что для операторов $A(t)$ с областью определения $D[A(t)]=D$, не зависящей от $t$, в качестве $E$ следует взять

$$
E=\left\{u \in D \mid\|u\|_{E}=\|A(0) u\|\right\} .
$$

Для описания примера операторов $A(t)$ с переменной областью определения, удовлетворяюших перечисленным вьше условиям, рассмотрим пространство $H=L_{2}(\Omega)$, где $\Omega \subset \mathbb{R}^{n}$ - ограниченная область с гладкой границей $\partial \Omega$. Пусть $A(t)$ при каждом $t \in[0, T]$ является эллиптическим самосопряженным оператором, порожденным в пространстве $L_{2}(\Omega)$ симметричньм дифференциальньм выражением второго порядка

$$
-\sum_{i, j=1}^{n} \frac{\partial}{\partial x_{i}}\left(a_{i j}(t, x) \frac{\partial u}{\partial x_{j}}\right)+a(t, x) u
$$

и третьим краевым условием на $\partial \Omega$

$$
\sum_{i, j=1}^{n} a_{i j}(t, x) \cos \left(\nu, x_{i}\right) \frac{\partial u}{\partial x_{j}}+\sigma(t, x) u=0
$$

где $\nu$ - единичньй вектор внешней нормали к $\partial \Omega$. Коэффициенты $a_{i j}(t, x), a(t, x), \sigma(t, x)$ предполагаются достаточно гладкими. Для таких операторов (см. [2, с. 384], [3, с. 249]) $D[A(t)]$ состоит из функций пространства $W_{2}^{2}(\Omega)$, удовлетворяюших краевому условию $(3), D_{1 / 2}=W_{2}^{1}(\Omega)$, причем нормы пространств $V$ и $W_{2}^{1}(\Omega)$ эквивалентны. Положим $E=W_{2}^{2}(\Omega)$, тогда (см. $[1$, с. 56$\left.]\right)$

$$
[E, H]_{1 / 2}=\left[W_{2}^{2}(\Omega), L_{2}(\Omega)\right]_{1 / 2}=W_{2}^{1}(\Omega) .
$$

Считаем, что операторы $B(t)$ линейны при всех $t \in[0, T]$, области определения $D[B(t)] \supset D_{1 / 2}$, функция $B(t) u$ для $u \in D_{1 / 2}$ дифференцируема в $H$ по $t$, так что $\left\|B^{\prime}(t) u\right\| \leqslant c\left\|A^{1 / 2}(0) u\right\|$. Отметим, что отсюда следует оценка $\left\|B(t) A^{-1 / 2}(0)\right\| \leqslant b<\infty$. 
Функция $f(t)$ со значениями в $H$ предполагается непрерьвной на $[0, T]$. Обозначим через $V^{\prime}$ пространство, двойственное $V$ по отношению к $H$. Пусть функция $f(t)$ со значениями в $V^{\prime}$ сильно дифференцируема на $V$ по $t \in[0, T]$, т.е. существует $f^{\prime}(t) \in V^{\prime}$ такая, что для всех $v \in V$ выполняется

$$
\frac{d}{d t}(f(t), v)=\left(f^{\prime}(t), v\right)
$$

где под $\left(f^{\prime}(t), v\right)$ понимается значение функционала $f^{\prime}(t)$ на элементе $v$. Кроме того, считаем $f^{\prime}(t) \in L_{2}\left(0, T ; V^{\prime}\right)$.

Наконец, пусть $u^{0} \in D[A(0)]$.

В [4] установлено, что в принятых предположениях задача (1) однозначно разрешима и решение $u(t)$ такое, что

1) $u^{\prime}(t), A(t) u(t) \in C([0, T], H) ; u^{\prime}(t) \in L_{2}(0, T ; V)$;

2) функция $u^{\prime}(t)$ со значениями в $V^{\prime}$ при почти всех $t \in[0, T]$ сильно дифференцируема на $V$ и $u^{\prime \prime}(t) \in L_{2}\left(0, T ; V^{\prime}\right)$;

3) удовлетворяет уравнению и начальному условию;

4) справедлива оценка

$$
\max _{0 \leqslant t \leqslant T}\left\|u^{\prime}(t)\right\|^{2}+\max _{0 \leqslant t \leqslant T}\|A(t) u(t)\|^{2}+\int_{0}^{T}\left(\left\|u^{\prime}(t)\right\|_{V}^{2}+\left\|u^{\prime \prime}(t)\right\|_{V^{\prime}}^{2}\right) d t \leqslant M P^{2}\left(u^{0}, f\right)
$$

где константа $M$ не зависит от $u^{0}$ и $f(t)$, a

$$
P\left(u^{0}, f\right)=\left(\left\|A(0) u^{0}\right\|^{2}+\max _{0 \leqslant t \leqslant T}\|f(t)\|^{2}+\int_{0}^{T}\left\|f^{\prime}(t)\right\|_{V^{\prime}}^{2} d t\right)^{1 / 2} .
$$

Перейдем к описанию проекционно-разностного метода приближенного решения задачи (1). Пусть $V_{h}$ ( $h$ - положительный параметр) - произвольное конечномерное подпространство пространства $V$. Через $A_{h}(t)$ для $t \in[0, T]$ обозначим действующий в $V_{h}$ самосопряженньй положительно определенньй оператор, заданньй для произвольных $u_{h}, v_{h} \in V_{h}$ соотношением

$$
\left(A_{h}(t) u_{h}, v_{h}\right)=a\left(t, u_{h}, v_{h}\right) .
$$

Задаче (1) сопоставим разностную задачу в $V_{h}$ :

$$
\left(u_{k}-u_{k-1}\right) \tau^{-1}+A_{h}\left(t_{k}\right) u_{k}+P_{h} B\left(t_{k}\right) u_{k}=P_{h} f\left(t_{k}\right)
$$

где $k=1,2, \ldots, N$ ( $N$ - натуральное число), элемент $u_{0}=u_{0}^{h} \in V_{h}$ считается заданным, $\tau N=T, t_{k}=k \tau$, оператор $P_{h}$ является ортогональньг проектором в пространстве $H$ на $V_{h}$.

Рассматриваемьй в настоящей работе класс гладких решений задачи (1) позволил впервые получить эффективные коэрцитивные в сильных нормах оценки погрешности проекционно-разностного метода. Эти оценки дают сходимость в сильных нормах приближенных решений к точному для произвольной предельно плотной в пространстве $V$ системы подпространств $\left\{V_{h}\right\}$. Кроме того, если известны аппроксимационные свойства подпространств $V_{h}$, то из этих оценок погрешности следуют точные по порядку 
апшроксимации числовые характеристики скорости сходимости, что иллюстрируется на подпространствах $V_{h}$ типа конечных элементов.

Для обобщенно разрешимого уравнения (1) подобные вопросы были рассмотрены в [5] и [6], где коэрцитивные оценки погрешности установлены в энергетической норме. При более жестких, чем в [5] и [6], условиях на подпространства $V_{h}$ энергетические оценки погрешности проекционно-разностного метода получены в [7], где рассматривалось параболическое уравнение второго порядка с краевым условием Дирихле.

Заметим, что оценки погрешности проекционно-разностного метода в сильных нормах даже в простейших случаях (см., например, [8, с. 205]) получены при гораздо больших требованиях на гладкость решения задачи (1), чем в настоящей работе.

Установим разрешимость задачи (6), т.е. обратимость в $V_{h}$ оператора

$$
I+\tau A_{h}\left(t_{k}\right)+\tau P_{h} B\left(t_{k}\right)=\left(I+\tau P_{h} B\left(t_{k}\right)\left(I+\tau A_{h}\left(t_{k}\right)\right)^{-1}\right)\left(I+\tau A_{h}\left(t_{k}\right)\right),
$$

где $k=1,2, \ldots, N$. Имеем оценку

$$
\left\|P_{h} B\left(t_{k}\right)\left(I+\tau A_{h}\left(t_{k}\right)\right)^{-1}\right\| \leqslant b\left\|A^{1 / 2}(0)\left(I+\tau A_{h}\left(t_{k}\right)\right)^{-1}\right\| .
$$

Из (2) для $v \in V$

$$
\delta\|v\|_{V}^{2} \leqslant\left\|A^{1 / 2}(t) v\right\|^{2} \leqslant M_{1}\|v\|_{V}^{2} .
$$

Кроме того, из (5) для $v_{h} \in V_{h}$ получим

$$
\left.\| A^{1 / 2}(t) v_{h}\right)\|=\| A_{h}^{1 / 2}(t) v_{h} \| .
$$

Таким образом,

$$
\left\|A^{1 / 2}(0)\left(I+\tau A_{h}\left(t_{k}\right)\right)^{-1}\right\| \leqslant \delta^{-1 / 2}\left\|A_{h}^{1 / 2}\left(t_{k}\right)\left(I+\tau A_{h}\left(t_{k}\right)\right)^{-1}\right\| .
$$

Из спектрального представления самосопряженного положительно определенного оператора $A_{h}\left(t_{k}\right)$ следует

$$
\left\|A_{h}^{1 / 2}\left(t_{k}\right)\left(I+\tau A_{h}\left(t_{k}\right)\right)^{-1}\right\| \leqslant \frac{1}{2} \tau^{-1 / 2} .
$$

Итак, для мальх $0<\tau \leqslant \tau_{0}$ доказана обратимость в $V_{h}$ оператора $I+\tau A_{h}\left(t_{k}\right)+\tau P_{h} B\left(t_{k}\right)$. Отметим, что $\tau_{0}$ не зависит от $V_{h}$.

Далее через $V(t)$ обозначим энергетическое пространство, порожденное оператором $A(t)$, т.е.

$$
V(t)=\left\{u \in D_{1 / 2} \mid\|u\|_{V(t)}=\left\|A^{1 / 2}(t) u\right\|\right\} .
$$

Пусть $Q_{h}(t)$ - ортогональный проектор в гильбертовом пространстве $V(t)$ на $V_{h}$. Обозначим $Q_{h}(0)=Q_{h}$.

Приведем две леммы, доказанные в [9].

Лемма 1. Для $u \in D_{1 / 2} u t \in[0, T]$ справедливы оченки

$$
\begin{gathered}
\left\|\left(I-Q_{h}(t)\right) u\right\|_{V(t)} \leqslant M_{1}^{1 / 2}\left\|\left(I-Q_{h}\right) u\right\|_{V}, \\
\left\|\left(I-Q_{h}(t)\right) u\right\| \leqslant M_{1}\left\|A^{1 / 2}(0)\left(I-Q_{h}\right) A^{-1}(t)\right\| \cdot\left\|\left(I-Q_{h}\right) u\right\|_{V} .
\end{gathered}
$$


Лемма 2. Oператор $Q_{h}(t)$ сильно дифференцируем в $V$ nо $t \in[0, T] u$ для $u \in V$

$$
\left\|Q_{h}^{\prime}(t) u\right\|_{V} \leqslant M_{1}^{1 / 2} M_{2} \delta^{-3 / 2}\left\|\left(I-Q_{h}\right) u\right\|_{V}
$$

\section{2. Оценки погрешности.}

Tеорема 1. Пусть $z_{k}=u\left(t_{k}\right)-u_{k}$, әде $k=1,2, \ldots, N, u(t)-$ решение задачи (1), а $u_{k}$ - решение задачи (6). Тогда выполняется оченка

$$
\begin{aligned}
\sum_{k=1}^{N}\left(\left\|\left(z_{k}-z_{k-1}\right) \tau^{-1}\right\|^{2} \tau+\left\|z_{k}-z_{k-1}\right\|_{V}^{2}\right)+\max _{1 \leqslant k \leqslant N}\left\|z_{k}\right\|_{V}^{2} \\
\quad \leqslant\left(\left\|Q_{h} u^{0}-u_{0}^{h}\right\|_{V}^{2}+\tau P^{2}\left(u^{0}, f\right)+\max _{0 \leqslant t \leqslant T}\left\|\left(I-Q_{h}\right) u(t)\right\|_{V}^{2}\right. \\
\left.\quad+\int_{0}^{T}\left\|\left(I-Q_{h}(t)\right) u^{\prime}(t)\right\|^{2} d t\right)
\end{aligned}
$$

әде $P\left(u^{0}, f\right)$ определена в оченке $(4)$, а константа $M$ не зависит от $u^{0}, u_{0}^{h}, f(t)$, $\tau u V_{h}$.

ДокАЗАТЕЛЬСТво. Определим функцию $w_{h}(t)=Q_{h}(t) u(t)$. Отметим свойство

$$
P_{h} A(t) u(t)=A_{h}(t) w_{h}(t)
$$

которое следует из (5). Обозначим $z_{k}^{h}=w_{h}\left(t_{k}\right)-u_{k} \in V_{h}$. С учетом (11) нетрудно установить тождество

$\left(z_{k}^{h}-z_{k-1}^{h}\right) \tau^{-1}+A_{h}\left(t_{k}\right) z_{k}^{h}=P_{h} B\left(t_{k}\right)\left(u_{k}-u\left(t_{k}\right)\right)+P_{h}\left(\left(w_{h}\left(t_{k}\right)-w_{h}\left(t_{k-1}\right)\right) \tau^{-1}-u^{\prime}\left(t_{k}\right)\right)$.

Умножим (12) скалярно в $H$ на $\left(z_{k}^{h}-z_{k-1}^{h}\right) \tau^{-1}$. При этом появится слагаемое

$$
\begin{aligned}
\left(A_{h}\left(t_{k}\right) z_{k}^{h},\left(z_{k}^{h}-z_{k-1}^{h}\right) \tau^{-1}\right)= & \frac{1}{\tau} \int_{t_{k-1}}^{t_{k}} a\left(t, z_{k}^{h},\left(z_{k}^{h}-z_{k-1}^{h}\right) \tau^{-1}\right) d t \\
& +\frac{1}{\tau} \int_{t_{k-1}}^{t_{k}}\left(\int_{t}^{t_{k}} \frac{\partial}{\partial s} a\left(s, z_{k}^{h},\left(z_{k}^{h}-z_{k-1}^{h}\right) \tau^{-1}\right) d s\right) d t
\end{aligned}
$$

Преобразуем также

$$
\begin{gathered}
\left(\left(w_{h}\left(t_{k}\right)-w_{h}\left(t_{k-1}\right)\right) \tau^{-1}-u^{\prime}\left(t_{k}\right),\left(z_{k}^{h}-z_{k-1}^{h}\right) \tau^{-1}\right) \\
=\frac{1}{\tau} \int_{t_{k-1}}^{t_{k}}\left(u^{\prime}(t)-u^{\prime}\left(t_{k}\right),\left(z_{k}^{h}-z_{k-1}^{h}\right) \tau^{-1}\right) d t \\
\quad+\frac{1}{\tau} \int_{t_{k-1}}^{t_{k}}\left(w_{h}^{\prime}(t)-u^{\prime}(t),\left(z_{k}^{h}-z_{k-1}^{h}\right) \tau^{-1}\right) d t
\end{gathered}
$$


Слагаемое $\left(B\left(t_{k}\right)\left(u_{k}-u\left(t_{k}\right)\right),\left(z_{k}^{h}-z_{k-1}^{h}\right) \tau^{-1}\right)$ разобьем на два. В результате получим

$$
\begin{aligned}
& \left\|\left(z_{k}^{h}-z_{k-1}^{h}\right) \tau^{-1}\right\|^{2}+\frac{1}{\tau} \int_{t_{k-1}}^{t_{k}} a\left(t, z_{k}^{h},\left(z_{k}^{h}-z_{k-1}^{h}\right) \tau^{-1}\right) d t \\
& +\frac{1}{\tau} \int_{t_{k-1}}^{t_{k}}\left(\int_{t}^{t_{k}} \frac{\partial}{\partial s} a\left(s, z_{k}^{h},\left(z_{k}^{h}-z_{k-1}^{h}\right) \tau^{-1}\right) d s\right) d t \\
& =\left(B\left(t_{k}\right)\left(w_{h}\left(t_{k}\right)-u\left(t_{k}\right)\right),\left(z_{k}^{h}-z_{k-1}^{h}\right) \tau^{-1}\right)-\left(B\left(t_{k}\right) z_{k}^{h},\left(z_{k}^{h}-z_{k-1}^{h}\right) \tau^{-1}\right) \\
& \quad+\frac{1}{\tau} \int_{t_{k-1}}^{t_{k}}\left(u^{\prime}(t)-u^{\prime}\left(t_{k}\right),\left(z_{k}^{h}-z_{k-1}^{h}\right) \tau^{-1}\right) d t \\
& \quad+\frac{1}{\tau} \int_{t_{k-1}}^{t_{k}}\left(w_{h}^{\prime}(t)-u^{\prime}(t),\left(z_{k}^{h}-z_{k-1}^{h}\right) \tau^{-1}\right) d t .
\end{aligned}
$$

Рассмотрим здесь

$$
\begin{aligned}
& \frac{2}{\tau} \int_{t_{k-1}}^{t_{k}} \operatorname{Re} a\left(t, z_{k}^{h},\left(z_{k}^{h}-z_{k-1}^{h}\right) \tau^{-1}\right) d t \\
& \quad=\frac{1}{\tau}\left\|A^{1 / 2}\left(t_{k}\right) z_{k}^{h}\right\|^{2}-\frac{1}{\tau}\left\|A^{1 / 2}\left(t_{k-1}\right) z_{k-1}^{h}\right\|^{2}+\frac{1}{\tau^{2}} \int_{t_{k-1}}^{t_{k}}\left\|A^{1 / 2}(t)\left(z_{k}^{h}-z_{k-1}^{h}\right)\right\|^{2} d t \\
& \quad+\frac{1}{\tau^{2}} \int_{t_{k-1}}^{t_{k}}\left(a\left(t, z_{k}^{h}, z_{k}^{h}\right)-a\left(t_{k}, z_{k}^{h}, z_{k}^{h}\right)\right) d t \\
& \quad+\frac{1}{\tau^{2}} \int_{t_{k-1}}^{t_{k}}\left(a\left(t_{k-1}, z_{k-1}^{h}, z_{k-1}^{h}\right)-a\left(t, z_{k-1}^{h}, z_{k-1}^{h}\right)\right) d t .
\end{aligned}
$$

Последнее тождество позволяет записать (13) в виде

$$
\begin{aligned}
2\left\|\left(z_{k}^{h}-z_{k-1}^{h}\right) \tau^{-1}\right\|^{2}+\frac{1}{\tau^{2}} \int_{t_{k-1}}^{t_{k}}\left\|A^{1 / 2}(t)\left(z_{k}^{h}-z_{k-1}^{h}\right)\right\|^{2} d t \\
+\frac{1}{\tau}\left\|A^{1 / 2}\left(t_{k}\right) z_{k}^{h}\right\|^{2}-\frac{1}{\tau}\left\|A^{1 / 2}\left(t_{k-1}\right) z_{k-1}^{h}\right\|^{2} \\
=\frac{1}{\tau^{2}} \int_{t_{k-1}}^{t_{k}}\left(a\left(t_{k}, z_{k}^{h}, z_{k}^{h}\right)-a\left(t, z_{k}^{h}, z_{k}^{h}\right)\right) d t \\
\quad+\frac{1}{\tau^{2}} \int_{t_{k-1}}^{t_{k}}\left(a\left(t, z_{k-1}^{h}, z_{k-1}^{h}\right)-a\left(t_{k-1}, z_{k-1}^{h}, z_{k-1}^{h}\right)\right) d t \\
\quad-\frac{2}{\tau} \int_{t_{k-1}}^{t_{k}}\left(\int_{t}^{t_{k}} \operatorname{Re} \frac{\partial}{\partial s} a\left(s, z_{k}^{h},\left(z_{k}^{h}-z_{k-1}^{h}\right) \tau^{-1}\right) d s\right) d t \\
\quad+2 \operatorname{Re}\left(B\left(t_{k}\right)\left(w_{h}\left(t_{k}\right)-u\left(t_{k}\right)\right),\left(z_{k}^{h}-z_{k-1}^{h}\right) \tau^{-1}\right)-2 \operatorname{Re}\left(B\left(t_{k}\right) z_{k}^{h},\left(z_{k}^{h}-z_{k-1}^{h}\right) \tau^{-1}\right) \\
\quad+\frac{2}{\tau} \int_{t_{k-1}}^{t_{k}} \operatorname{Re}\left(u^{\prime}(t)-u^{\prime}\left(t_{k}\right),\left(z_{k}^{h}-z_{k-1}^{h}\right) \tau^{-1}\right) d t \\
\quad+\frac{2}{\tau} \int_{t_{k-1}}^{t_{k}} \operatorname{Re}\left(w_{h}^{\prime}(t)-u^{\prime}(t),\left(z_{k}^{h}-z_{k-1}^{h}\right) \tau^{-1}\right) d t=\sum_{i=1}^{7} J_{i} .
\end{aligned}
$$


Оценим слагаемые $J_{i}$ в правой части (14):

$J_{1} \leqslant M_{2}\left\|z_{k}^{h}\right\|_{V}^{2}, \quad J_{2} \leqslant M_{2}\left\|z_{k-1}^{h}\right\|_{V}^{2}, \quad J_{3} \leqslant \frac{M_{2}}{\varepsilon_{1}}\left\|z_{k}^{h}\right\|_{V}^{2}+M_{2} T \varepsilon_{1} \frac{1}{\tau}\left\|z_{k}^{h}-z_{k-1}^{h}\right\|_{V}^{2}$

$$
\begin{gathered}
J_{4} \leqslant \frac{b}{\varepsilon_{2}} \max _{0 \leqslant t \leqslant T}\left\|w_{h}(t)-u(t)\right\|_{V}^{2}+b \varepsilon_{2}\left\|\left(z_{k}^{h}-z_{k-1}^{h}\right) \tau^{-1}\right\|^{2}, \\
J_{5} \leqslant \frac{b}{\varepsilon_{3}}\left\|z_{k}^{h}\right\|_{V}^{2}+b \varepsilon_{3}\left\|\left(z_{k}^{h}-z_{k-1}^{h}\right) \tau^{-1}\right\|^{2} .
\end{gathered}
$$

При оценке слагаемого $J_{6}$ вспомним, что $u^{\prime \prime}(t) \in L_{2}\left(0, T ; V^{\prime}\right)$ :

$$
\begin{aligned}
J_{6} & =\frac{2}{\tau^{2}} \int_{t_{k-1}}^{t_{k}}\left(\int_{t_{k}}^{t} \operatorname{Re}\left(u^{\prime \prime}(s), z_{k}^{h}-z_{k-1}^{h}\right) d s\right) d t \\
& \leqslant \frac{1}{\varepsilon_{4}} \int_{t_{k-1}}^{t_{k}}\left\|u^{\prime \prime}(s)\right\|_{V^{\prime}}^{2} d s+\varepsilon_{4} \frac{1}{\tau}\left\|z_{k}^{h}-z_{k-1}^{h}\right\|_{V}^{2}
\end{aligned}
$$

Переходим к последнему слагаемому:

$$
J_{7} \leqslant \frac{1}{\varepsilon_{5} \tau} \int_{t_{k-1}}^{t_{k}}\left\|w_{h}^{\prime}(t)-u^{\prime}(t)\right\|^{2} d t+\varepsilon_{5}\left\|\left(z_{k}^{h}-z_{k-1}^{h}\right) \tau^{-1}\right\|^{2} .
$$

Наконец, оценим снизу левую часть тождества (14):

$$
\frac{1}{\tau^{2}} \int_{t_{k-1}}^{t_{k}}\left\|A^{1 / 2}(t)\left(z_{k}^{h}-z_{k-1}^{h}\right)\right\|^{2} d t \geqslant \frac{\delta}{\tau}\left\|z_{k}^{h}-z_{k-1}^{h}\right\|_{V}^{2}
$$

Положив теперь $M_{2} T \varepsilon_{1}=\varepsilon_{4}=\delta / 4, b \varepsilon_{2}=b \varepsilon_{3}=\varepsilon_{5}=1 / 3$, из (14) получим неравенство

$$
\begin{aligned}
& \left\|\left(z_{k}^{h}-z_{k-1}^{h}\right) \tau^{-1}\right\|^{2}+\frac{1}{\tau}\left\|z_{k}^{h}-z_{k-1}^{h}\right\|_{V}^{2}+\frac{1}{\tau}\left\|A^{1 / 2}\left(t_{k}\right) z_{k}^{h}\right\|^{2}-\frac{1}{\tau}\left\|A^{1 / 2}\left(t_{k-1}\right) z_{k-1}^{h}\right\|^{2} \\
& \leqslant c_{1} \max _{0 \leqslant t \leqslant T}\left\|w_{h}(t)-u(t)\right\|_{V}^{2}+c_{2} \frac{1}{\tau} \int_{t_{k-1}}^{t_{k}}\left\|w_{h}^{\prime}(t)-u^{\prime}(t)\right\|^{2} d t \\
& \quad+c_{3} \int_{t_{k-1}}^{t_{k}}\left\|u^{\prime \prime}(t)\right\|_{V^{\prime}}^{2} d t+c_{4}\left\|z_{k}^{h}\right\|_{V}^{2}+c_{5}\left\|z_{k-1}^{h}\right\|_{V}^{2}
\end{aligned}
$$

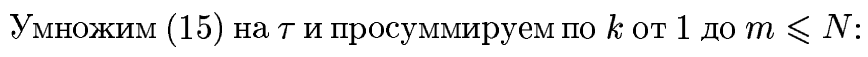

$$
\begin{aligned}
& \sum_{k=1}^{m}\left(\left\|\left(z_{k}^{h}-z_{k-1}^{h}\right) \tau^{-1}\right\| \tau+\left\|z_{k}^{h}-z_{k-1}^{h}\right\|_{V}^{2}\right)+\left\|A^{1 / 2}\left(t_{m}\right) z_{m}^{h}\right\|^{2}-\left\|A^{1 / 2}(0) z_{0}^{h}\right\|^{2} \\
& \quad \leqslant c_{1} \max _{0 \leqslant t \leqslant T}\left\|w_{h}(t)-u(t)\right\|_{V}^{2}+c_{2} \int_{0}^{T}\left\|w_{h}^{\prime}(t)-u^{\prime}(t)\right\|^{2} d t \\
& \quad+c_{3} \tau \int_{0}^{T}\left\|u^{\prime \prime}(t)\right\|_{V^{\prime}}^{2} d t+c_{4} \sum_{k=1}^{m}\left\|z_{k}^{h}\right\|_{V}^{2} \tau+c_{5}\left\|z_{0}^{h}\right\|_{V}^{2}
\end{aligned}
$$


Выделяя в (16) суммарное неравенство для $\left\|z_{m}^{h}\right\|_{V}^{2}$, а именно:

$$
\begin{aligned}
\left\|z_{m}^{h}\right\|_{V}^{2} \leqslant & c_{1}\left(\left\|Q_{h} u^{0}-u_{0}^{h}\right\|_{V}^{2}+\max _{0 \leqslant t \leqslant T}\left\|w_{h}(t)-u(t)\right\|_{V}^{2}\right. \\
& \left.+\int_{0}^{T}\left\|w_{h}^{\prime}(t)-u^{\prime}(t)\right\|^{2} d t+\tau \int_{0}^{T}\left\|u^{\prime \prime}(t)\right\|_{V^{\prime}}^{2} d t\right)+c_{2} \sum_{k=1}^{m}\left\|z_{k}^{h}\right\|_{V}^{2} \tau,
\end{aligned}
$$

получим стандартными рассуждениями оценку

$$
\begin{aligned}
& \sum_{k=1}^{N}\left(\left\|\left(z_{k}^{h}-z_{k-1}^{h}\right) \tau^{-1}\right\|^{2} \tau+\left\|z_{k}^{h}-z_{k-1}^{h}\right\|_{V}^{2}\right)+\max _{1 \leqslant k \leqslant N}\left\|z_{k}^{h}\right\|_{V}^{2} \\
& \leqslant M\left(\left\|Q_{h} u^{0}-u_{0}^{h}\right\|_{V}^{2}+\tau \int_{0}^{T}\left\|u^{\prime \prime}(t)\right\|_{V^{\prime}}^{2} d t\right. \\
& \left.\quad+\max _{0 \leqslant t \leqslant T}\left\|w_{h}(t)-u(t)\right\|_{V}^{2}+\int_{0}^{T}\left\|w_{h}^{\prime}(t)-u^{\prime}(t)\right\|^{2} d t\right) .
\end{aligned}
$$

Оценку (17) преобразуем к виду, которьй позволил бы оценить погрешность $z_{k}=$ $\left(u\left(t_{k}\right)-w_{h}\left(t_{k}\right)\right)+z_{k}^{h}$. Очевидно, что

$$
\max _{1 \leqslant k \leqslant N}\left\|z_{k}\right\|_{V}^{2} \leqslant 2 \max _{1 \leqslant k \leqslant N}\left\|z_{k}^{h}\right\|_{V}^{2}+2 \max _{0 \leqslant t \leqslant T}\left\|u(t)-w_{h}(t)\right\|_{V}^{2} .
$$

Из соотношения

$$
z_{k}-z_{k-1}=\left(z_{k}^{h}-z_{k-1}^{h}\right)+\int_{t_{k-1}}^{t_{k}}\left(u^{\prime}(t)-w_{h}^{\prime}(t)\right) d t
$$

следует оценка

$$
\begin{aligned}
& \sum_{k=1}^{N}\left(\left\|\left(z_{k}-z_{k-1}\right) \tau^{-1}\right\|^{2} \tau+\left\|z_{k}-z_{k-1}\right\|_{V}^{2}\right) \\
& \quad \leqslant 2 \sum_{k=1}^{N}\left(\left\|\left(z_{k}^{h}-z_{k-1}^{h}\right) \tau^{-1}\right\|^{2} \tau+\left\|z_{k}^{h}-z_{k-1}^{h}\right\|_{V}^{2}\right) \\
& \quad+2 \int_{0}^{T}\left\|u^{\prime}(t)-w_{h}^{\prime}(t)\right\|^{2} d t+2 \tau \int_{0}^{T}\left\|u^{\prime}(t)-w_{h}^{\prime}(t)\right\|_{V}^{2} d t
\end{aligned}
$$

Заметим, что при почти всех $t \in[0, T]$

$$
w_{h}^{\prime}(t)=Q_{h}(t) u^{\prime}(t)+Q_{h}^{\prime}(t) u(t) .
$$

Следовательно, учитьвая оценки (7), (9) и (4), получим

$$
\begin{aligned}
\int_{0}^{T}\left\|u^{\prime}(t)-w_{h}^{\prime}(t)\right\|^{2} d t & \leqslant c_{1} \max _{0 \leqslant t \leqslant T}\left\|\left(I-Q_{h}\right) u(t)\right\|_{V}^{2}+c_{2} \int_{0}^{T}\left\|\left(I-Q_{h}(t)\right) u^{\prime}(t)\right\|^{2} d t \\
\int_{0}^{T}\left\|u^{\prime}(t)-w_{h}^{\prime}(t)\right\|_{V}^{2} d t & \leqslant c_{1} \max _{0 \leqslant t \leqslant T}\left\|\left(I-Q_{h}\right) u(t)\right\|_{V}^{2}+c_{2} \int_{0}^{T}\left\|\left(I-Q_{h}\right) u^{\prime}(t)\right\|_{V}^{2} d t \\
& \leqslant c\left(\max _{0 \leqslant t \leqslant T}\|A(t) u(t)\|^{2}+\int_{0}^{T}\left\|u^{\prime}(t)\right\|_{V}^{2} d t\right) \leqslant M P^{2}\left(u^{0}, f\right) .
\end{aligned}
$$


Оценка (10) следует теперь из оценок (18)-(20), (7) и (4).

Обратим внимание, что в левой части оценки (10)

$$
\begin{aligned}
& \sum_{k=1}^{N}\left(\left\|\left(z_{k}-z_{k-1}\right) \tau^{-1}\right\|^{2} \tau+\left\|z_{k}-z_{k-1}\right\|_{V}^{2}\right) \\
& \quad=\sum_{k=1}^{N}\left\|\frac{1}{\tau} \int_{t_{k-1}}^{t_{k}}\left(u^{\prime}(t)-\frac{u_{k}-u_{k-1}}{\tau}\right) d t\right\|^{2} \tau+\sum_{k=1}^{N}\left\|\int_{t_{k-1}}^{t_{k}}\left(u^{\prime}(t)-\frac{u_{k}-u_{k-1}}{\tau}\right) d t\right\|_{V}^{2} .
\end{aligned}
$$

Покажем эффективность оценки (10). Предположим, что задана последовательность конечномерных подпространств $\left\{V_{h}\right\}$, предельно плотная в $V$ при $h \rightarrow 0$, иными словами, $\left\|\left(I-Q_{h}\right) v\right\|_{V} \rightarrow 0$ при $h \rightarrow 0$ для любого $v \in V$. Пусть также $\left\|Q_{h} u^{0}-u_{0}^{h}\right\|_{V} \rightarrow 0$. В таком случае при $h \rightarrow 0$ и $\tau \rightarrow 0$ из (10) следует

$$
\sum_{k=1}^{N}\left(\left\|\left(z_{k}-z_{k-1}\right) \tau^{-1}\right\|^{2} \tau+\left\|z_{k}-z_{k-1}\right\|_{V}^{2}\right)+\max _{1 \leqslant k \leqslant N}\left\|z_{k}\right\|_{V}^{2} \rightarrow 0,
$$

так как $u(t) \in C([0, T], V)$ и $u^{\prime}(t) \in L_{2}(0, T ; V)$.

Оценка (10) позволяет также получать числовые характеристики скорости сходимости. Предположим, что подпространства $V_{h}$ обладают следующим аппроксимационным свойством, типичным (см., например, [10, с. 128]) для метода конечных элементов:

$$
\left\|\left(I-Q_{h}\right) v\right\|_{V} \leqslant c h\|v\|_{E}, \quad v \in E,
$$

где константа $c$ не зависит от $v$ и $h$. Из (21) следуют оценки

$$
\left\|\left(I-Q_{h}\right) v\right\|_{V} \leqslant c d h\|A(t) v\|, \quad v \in D[A(t)], \quad\left\|A^{1 / 2}(0)\left(I-Q_{h}\right) A^{-1}(t)\right\| \leqslant c d h .
$$

В результате из оценки (10), условия $(21)$, оценок $(8),(22)$ и (4) получим для погрешности $z_{k}=u\left(t_{k}\right)-u_{k}$ оценку

$$
\begin{aligned}
& \sum_{k=1}^{N}\left(\left\|\left(z_{k}-z_{k-1}\right) \tau^{-1}\right\|^{2} \tau+\left\|z_{k}-z_{k-1}\right\|_{V}^{2}\right)+\max _{1 \leqslant k \leqslant N}\left\|z_{k}\right\|_{V}^{2} \\
& \quad \leqslant M\left(\left\|Q_{h} u^{0}-u_{0}^{h}\right\|_{V}^{2}+\left(\tau+h^{2}\right) P^{2}\left(u^{0}, f\right)\right) .
\end{aligned}
$$

Докажем теперь, что порядок сходимости к 0, установленньй в оценках (10) и (23), для более слабой нормы можно увеличить.

Tеорема 2. Пусть $z_{k}=u\left(t_{k}\right)-u_{k}$, әде $k=1,2, \ldots, N, u(t)$-решение задачи (1), а $u_{k}$ - решение задачи (6). Тогда выполняется оченка

$$
\begin{aligned}
\max _{1 \leqslant k \leqslant N} & \left\|z_{k}\right\|^{2}+\sum_{k=1}^{N}\left(\left\|z_{k}\right\|_{V}^{2} \tau+\left\|z_{k}-z_{k-1}\right\|^{2}\right) \\
\leqslant & M\left(\left\|u^{0}-u_{0}^{h}\right\|^{2}+\tau^{2} P^{2}\left(u^{0}, f\right)+\max _{0 \leqslant t \leqslant T}\left\|\left(I-Q_{h}\right) u(t)\right\|_{V}^{2}\right. \\
& \left.+\left(P\left(u^{0}, f\right)+\left\|u_{0}^{h}\right\|_{V}\right) \max _{0 \leqslant t \leqslant T}\left\|\left(I-Q_{h}(t)\right) u(t)\right\|\right)
\end{aligned}
$$

әде $P\left(u^{0}, f\right)$ определена в оченке (4), а константа $M$ не зависит от $u^{0}, u_{0}^{h}, f(t)$, $\tau u V_{h}$. 
ДоказАтеЛЬСтво. Рассмотрим тождество

$$
\begin{aligned}
& \left(\left(z_{k}-z_{k-1}\right) \tau^{-1}, z_{k}\right)+\left\|A_{h}^{1 / 2}\left(t_{k}\right)\left(w_{h}\left(t_{k}\right)-u_{k}\right)\right\|^{2} \\
& \quad=\left(\left(z_{k}-z_{k-1}\right) \tau^{-1}, u\left(t_{k}\right)-w_{h}\left(t_{k}\right)\right)+\frac{1}{\tau} \int_{t_{k-1}}^{t_{k}}\left(u^{\prime}(t)-u^{\prime}\left(t_{k}\right), w_{h}\left(t_{k}\right)-u_{k}\right) d t \\
& \quad-\left(B\left(t_{k}\right) z_{k}, w_{h}\left(t_{k}\right)-u_{k}\right),
\end{aligned}
$$

из которого получим

$$
\begin{aligned}
\frac{1}{\tau}\left\|z_{k}\right\|^{2}-\frac{1}{\tau}\left\|z_{k-1}\right\|^{2}+\frac{1}{\tau}\left\|z_{k}-z_{k-1}\right\|^{2}+2\left\|A^{1 / 2}\left(t_{k}\right)\left(w_{h}\left(t_{k}\right)-u_{k}\right)\right\|^{2} \\
=2 \operatorname{Re}\left(\left(z_{k}-z_{k-1}\right) \tau^{-1}, u\left(t_{k}\right)-w_{h}\left(t_{k}\right)\right)+\frac{2}{\tau} \int_{t_{k-1}}^{t_{k}} \operatorname{Re}\left(u^{\prime}(t)-u^{\prime}\left(t_{k}\right), w_{h}\left(t_{k}\right)-u_{k}\right) d t \\
\quad-2 \operatorname{Re}\left(B\left(t_{k}\right) z_{k}, w_{h}\left(t_{k}\right)-u_{k}\right)=\sum_{i=1}^{3} J_{i} .
\end{aligned}
$$

Оценим слагаемые $J_{i}$ в правой части (25):

$$
J_{1} \leqslant 2\left\|\left(z_{k}-z_{k-1}\right) \tau^{-1}\right\| \cdot\left\|\left(I-Q_{h}\left(t_{k}\right)\right) u\left(t_{k}\right)\right\| .
$$

Слагаемое $J_{2}$ оценим подобно слагаемому $J_{6}$ в (14):

$$
\begin{aligned}
J_{2} & =\frac{2}{\tau} \int_{t_{k-1}}^{t_{k}}\left(\int_{t_{k}}^{t} \operatorname{Re}\left(u^{\prime \prime}(s), w_{h}\left(t_{k}\right)-u_{k}\right) d s\right) d t \\
& \leqslant \frac{\tau}{\varepsilon_{1}} \int_{t_{k-1}}^{t_{k}}\left\|u^{\prime \prime}(s)\right\|_{V^{\prime}}^{2} d s+\varepsilon_{1}\left\|w_{h}\left(t_{k}\right)-u_{k}\right\|_{V}^{2} .
\end{aligned}
$$

Слагаемое $J_{3}$ разобьем на три:

$$
\begin{aligned}
J_{3}= & 2 \operatorname{Re}\left(B\left(t_{k}\right)\left(u_{k}-w_{h}\left(t_{k}\right)\right), w_{h}\left(t_{k}\right)-u\left(t_{k}\right)\right) \\
& +2 \operatorname{Re}\left(B\left(t_{k}\right)\left(u_{k}-w_{h}\left(t_{k}\right)\right), z_{k}\right)+2 \operatorname{Re}\left(B\left(t_{k}\right)\left(w_{h}\left(t_{k}\right)-u\left(t_{k}\right)\right), w_{h}\left(t_{k}\right)-u_{k}\right) .
\end{aligned}
$$

Отсюда следует оценка

$$
\begin{aligned}
J_{3} \leqslant & b^{2} \varepsilon_{2}\left\|u_{k}-w_{h}\left(t_{k}\right)\right\|_{V}^{2}+\frac{1}{\varepsilon^{2}}\left\|w_{h}\left(t_{k}\right)-u\left(t_{k}\right)\right\|_{V}^{2} \\
& +b^{2} \varepsilon_{3}\left\|u_{k}-w_{h}\left(t_{k}\right)\right\|_{V}^{2}+\frac{1}{\varepsilon_{3}}\left\|z_{k}\right\|^{2}+\frac{b^{2}}{\varepsilon_{4}}\left\|w_{h}\left(t_{k}\right)-u\left(t_{k}\right)\right\|_{V}^{2}+\varepsilon_{4}\left\|w_{h}\left(t_{k}\right)-u_{k}\right\|_{V}^{2} .
\end{aligned}
$$

Оценим сверху

$$
2\left\|A^{1 / 2}\left(t_{k}\right)\left(w_{h}\left(t_{k}\right)-u_{k}\right)\right\|^{2} \geqslant 2 \delta\left\|w_{h}\left(t_{k}\right)-u_{k}\right\|_{V}^{2} .
$$

Выбирая $\varepsilon_{1}=b^{2} \varepsilon_{2}=b^{2} \varepsilon_{3}=\varepsilon_{4}=\delta / 4$, получим из (25) неравенство

$$
\begin{gathered}
\frac{1}{\tau}\left\|z_{k}\right\|^{2}-\frac{1}{\tau}\left\|z_{k-1}\right\|^{2}+\frac{1}{\tau}\left\|z_{k}-z_{k-1}\right\|^{2}+\delta\left\|w_{h}\left(t_{k}\right)-u_{k}\right\|_{V}^{2} \\
\leqslant \frac{4 b^{2}}{\delta}\left\|z_{k}\right\|^{2}+2\left\|\left(z_{k}-z_{k-1}\right) \tau^{-1}\right\| \cdot\left\|\left(I-Q_{h}\left(t_{k}\right)\right) u\left(t_{k}\right)\right\| \\
+\frac{4 \tau}{\delta} \int_{t_{k-1}}^{t_{k}}\left\|u^{\prime \prime}(t)\right\|_{V^{\prime}}^{2} d t+\frac{8 b^{2}}{\delta}\left\|\left(I-Q_{h}\left(t_{k}\right)\right) u\left(t_{k}\right)\right\|_{V}^{2} .
\end{gathered}
$$




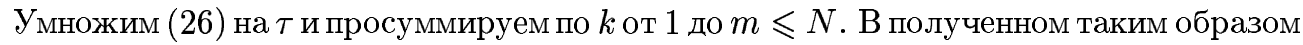
неравенстве выделим суммарное неравенство для $\left\|z_{m}\right\|^{2}$, а именно:

$$
\begin{aligned}
& \left\|z_{m}\right\|^{2} \leqslant c_{1} \sum_{k=1}^{m}\left\|z_{k}\right\|^{2} \tau+c_{2}\left(\sum_{k=1}^{N}\left\|\left(z_{k}-z_{k-1}\right) \tau^{-1}\right\|^{2} \tau\right)^{1 / 2} \max _{0 \leqslant t \leqslant T}\left\|\left(I-Q_{h}(t)\right) u(t)\right\| \\
& +c_{3} \tau^{2} \int_{0}^{T}\left\|u^{\prime \prime}(t)\right\|_{V^{\prime}}^{2} d t+c_{4} \max _{0 \leqslant t \leqslant T}\left\|\left(I-Q_{h}(t)\right) u(t)\right\|_{V^{\prime}}^{2} \text {. }
\end{aligned}
$$

В результате из $(27),(26)$ и (4) получим оценку

$$
\begin{aligned}
\max _{1 \leqslant k \leqslant N}\left\|z_{k}\right\|^{2}+\sum_{k=1}^{N}\left(\left\|z_{k}\right\|_{V}^{2} \tau+\left\|z_{k}-z_{k-1}\right\|^{2}\right) \\
\leqslant M\left(\left\|u^{0}-u_{0}^{h}\right\|^{2}+\tau^{2} P^{2}\left(u^{0}, f\right)+\max _{0 \leqslant t \leqslant T}\left\|\left(I-Q_{h}\right) u(t)\right\|_{V}^{2}\right. \\
\left.\quad+\left(\sum_{k=1}^{N}\left\|\left(z_{k}-z_{k-1}\right) \tau^{-1}\right\|^{2} \tau\right)^{1 / 2} \max _{0 \leqslant t \leqslant T}\left\|\left(I-Q_{h}(t)\right) u(t)\right\|\right) .
\end{aligned}
$$

Оценка (24) следует теперь из (28) и оценки

$$
\left(\sum_{k=1}^{N}\left\|\left(z_{k}-z_{k-1}\right) \tau^{-1}\right\|^{2} \tau\right)^{1 / 2} \leqslant M\left(P\left(u^{0}, f\right)+\left\|u_{0}^{h}\right\|_{V}\right)
$$

вытекающей из оценок (10) и (4).

Если, как и ранее, $\left\{V_{h}\right\}$ - предельно плотная в $V$ последовательность конечномерных подпространств, $\left\|u^{0}-u_{0}^{h}\right\| \rightarrow 0$ при $h \rightarrow 0$ и последовательность $\left\{\left\|u_{0}^{h}\right\|_{V}\right\}$, допустим, ограничена (хотя возможен даже некоторый рост), то из (24) при $h \rightarrow 0$ и $\tau \rightarrow 0$ следует

$$
\max _{1 \leqslant k \leqslant N}\left\|z_{k}\right\|^{2}+\sum_{k=1}^{N}\left(\left\|z_{k}\right\|_{V}^{2} \tau+\left\|z_{k}-z_{k-1}\right\|^{2}\right) \rightarrow 0
$$

Предположим, что подпространства $V_{h}$ обладают свойством $(21)$. Тогда из $(24)$, подобно оценке (23), получим

$$
\begin{aligned}
& \max _{1 \leqslant k \leqslant N}\left\|z_{k}\right\|^{2}+\sum_{k=1}^{N}\left(\left\|z_{k}\right\|_{V}^{2} \tau+\left\|z_{k}-z_{k-1}\right\|^{2}\right) \\
& \quad \leqslant M\left(\left\|u^{0}-u_{0}^{h}\right\|^{2}+\tau^{2} P^{2}\left(u^{0}, f\right)+h^{2}\left(P^{2}\left(u^{0}, f\right)+\left\|u_{0}^{h}\right\|_{V}^{2}\right)\right) .
\end{aligned}
$$

При более жестких условиях на подпространства $V_{h}$, функцию $f(t)$ и начальные условия $u_{0}^{h}$ в заметке [7] анонсирована энергетическая оценка погрешности того же порядка по $\tau$ и $h$, что и оценка (29). 


\section{СПИСОК ЦИТИРОВАННОЙ ЛИТЕРАТУРЫ}

[1] Лионс Ж.-Л., Мадженес Э. Неоднородные граничные задачи и их приложения. М.: Мир, 1971.

[2] Березанский Ю. М. Разложение по собственным функциям самосопряженных операторов. Киев: Наукова думка, 1965.

[3] С. Г. Крейн (ред. ) Функциональный анализ. Справочная математическая библиотека. 2-е изд. М.: Наука, 1972.

[4] Смагин В.В. О разрешимости абстрактного параболического уравнения с оператором, область определения которого зависит от времени // Дифференц. уравнения. 1996. Т. 32. № 5 . C. $711-712$.

[5] Смагин В. В. Коэрцитивные оценки погрешностей проекционного и проекционно-разностного методов для параболических уравнений // Матем. сб. 1994. Т. 185. № 11. С. 79-94.

[6] Смагин В. В. Коэрцитивные оценки погрешностей проекционно-разностного метода для абстрактного параболического уравнения с оператором, область определения которого зависит от времени // Сиб. матем. ж. 1996. Т. 37. № 2. С. 406-418.

[7] Злотник А. А. Оценки скорости сходимости в $V_{2}\left(Q_{T}\right)$ проекционно-разностных схем для параболических уравнений // Вестн. МГУ. Сер. 15. Вычислит. матем., киберн. 1980. № 1. C. $27-35$.

[8] Марчук Г.И., Агошков В.И. Введение в проекционно-сеточные методы. М.: Наука, 1981.

[9] Смагин В.В. Оценки погрешности полудискретных приближений по Галёркину для параболических уравнений с краевым условием типа Неймана // Изв. вузов. Матем. 1996.o․ C. $50-57$.

[10] Стренг Г., Фикс Дж. Теория метода конечных элементов. М.: Мир, 1977.

Воронежский государственный университет 